Published in final edited form as:

Spine J. 2020 January ; 20(1): 5-13. doi:10.1016/j.spinee.2019.01.014.

\title{
Laboratory Markers as Useful Prognostic Measures for Survival in Patients with Spinal Metastases
}

\author{
Andrew J. Schoenfeld, MD, MSc, \\ Department of Orthopaedic Surgery, Brigham and Women's Hospital, Harvard Medical School, \\ 75 Francis Street, Boston, MA 02115
}

Marco L. Ferrone, MD,

Department of Orthopaedic Surgery, Brigham and Women's Hospital, Harvard Medical School, 75 Francis Street, Boston, MA 02115

Peter G. Passias, MD,

New York Spine Institute, New York University Langone Medical Center, 761 Merrick Avenue, Westbury, NY 11590

Justin A. Blucher, MS,

Department of Orthopaedic Surgery, Brigham and Women's Hospital, Harvard Medical School, 75 Francis Street, Boston, MA 02115

\section{Lauren B. Barton, BS,}

Department of Orthopaedic Surgery, Brigham and Women's Hospital, Harvard Medical School, 75 Francis Street, Boston, MA 02115

John H. Shin, MD,

Department of Neurosurgery, Massachusetts General Hospital, 55 Fruit Street, Boston, MA 02214

\section{Mitchel B. Harris, MD,}

Department of Orthopaedic Surgery, Massachusetts General Hospital, 55 Fruit Street, Boston, MA 02214

\section{Joseph H. Schwab, MD, MS}

Department of Orthopaedic Surgery, Massachusetts General Hospital, 55 Fruit Street, Boston, MA 02214

Investigation performed at Brigham and Women's Hospital and Harvard Medical School, Boston, MA.

\section{Abstract}

\footnotetext{
Corresponding Author: ajschoen@neomed.edu.
}

Publisher's Disclaimer: This is a PDF file of an unedited manuscript that has been accepted for publication. As a service to our customers we are providing this early version of the manuscript. The manuscript will undergo copyediting, typesetting, and review of the resulting proof before it is published in its final citable form. Please note that during the production process errors may be discovered which could affect the content, and all legal disclaimers that apply to the journal pertain. 
BACKGROUND CONTEXT_Laboratory values have been found to be useful predictive measures of survival following surgery. The utility of laboratory values for prognosticating outcomes among patients with spinal metastases has not been studied.

PURPOSE-To determine the prognostic capacity of laboratory values at presentation including white blood cell (WBC) count, serum albumin and platelet-lymphocyte ratio (PLR) in patients with spinal metastases.

STUDY DESIGN—Retrospective review of records from two tertiary care centers (2005-2017).

PATIENT SAMPLE-Patients, aged 40-80, who received operative or non-operative management for spinal metastases.

OUTCOME MEASURES-Survival, complications or hospital readmissions within 90 days of treatment and a composite measure for treatment failure accounting for changes in ambulatory function and mortality at 6-months following presentation.

METHODS-Multivariable Cox proportional hazard regression analysis was used to analyze the relationship between laboratory values and length of survival, adjusting for confounders.

Multivariable logistic regression was used in analyses related to 6-month and 1-year mortality, complications, readmissions and treatment failure. A scoring rubric was developed based on the performance of laboratory values in the multivariable tests. Internal validation was performed using a bootstrap simulation that consisted of sampling with replacement and 1,000 replications.

RESULTS-We included 1,216 patients. Thirty-seven percent of patients received a surgical intervention and $63 \%$ were treated non-operatively. Median survival for the cohort as a whole was 255 days (inter-quartile range 93-642 days). PLR (HR 1.53; 95\% CI 1.29, 1.80; $<<0.001$ ) and albumin (HR 0.54; $95 \%$ CI $0.45,0.64 ; \mathrm{p}<0.001)$ were significantly associated with survival, while WBC count (HR 1.08; 95\% CI 0.86, 1.36; $\mathrm{p}=0.50$ ) was not associated with this outcome. Similar findings were encountered for 6-month and 1-year mortality as well as the composite measure for treatment failure. PLR and albumin performed well in our scoring rubric and findings were preserved in the bootstrapping validation.

CONCLUSIONS-Individuals with low serum albumin and elevated PLR should be advised regarding the impact of these laboratory markers on outcomes including survival, irrespective of treatments received. An effort should also be made to optimize nutrition and PLR, if practicable, prior to treatment to minimize the potential for development of adverse events.

\section{Keywords}

Spinal metastases; albumin; platelet-lymphocyte ratio; laboratory markers; prognostic models

\section{Introduction}

Recent investigations have reported that the prevalence of spinal metastases has increased over the last two decades, with approximately $7 / 10$ patients with metastatic disease found to have spinal involvement. ${ }^{1-4}$ The development of spinal metastases has been shown to have serious implications for physical function and survival. ${ }^{1,4-7}$ Patients with spinal metastases are reported to have a mortality rate as high as $50 \%$ within a calendar year of diagnosis. 2,4,5,7-12 While both operative and non-operative management have been found to be 
efficacious for spinal metastases $1,5-6,10,11$, initial decisions regarding care must balance the risks of these interventions with capacity to benefit from treatment. Both surgical and nonoperative treatment of spinal metastases carry risks of significant post-treatment morbidity which may affect survival. ${ }^{1,2,4,10,13,14}$

A critical component in decision making consists of the effective prognostication of survival, functional capacity, the development of complications and hospital encounters (e.g. readmissions). Understanding these parameters can help clinicians formulate treatment strategies and enable patients to make informed decisions regarding treatment that aligns best with their preferences and expectations for recovery. At the present time, however, there are limited prognostic utilities that apply to all patients with spinal metastases. Most predictive models are focused on individuals undergoing surgery $3,5,6,9,12$, or are specifically intended to inform aspects of surgical care. ${ }^{15,16}$ Relatively few schemes have been shown to be appropriate for patients receiving operative or non-operative care for spinal metastatic disease $\mathrm{e}^{14}$. Laboratory values, which are widely available in patients with cancer, have been found to be useful predictive measures in other areas of surgical and medical care. ${ }^{17-24}$ For example, a low white blood cell (WBC) count may herald increased risk of infection, while hypoalbuminemia is indicative of impaired nutritional status and capacity for wound healing. Both factors may be determinants of an individual's physiologic reserve, while the plateletlymphocyte ratio (PLR) has been described as a marker of inflammation. ${ }^{18-20}$ The utility of these laboratory values for prognosticating outcomes among patients with spinal metastases has not been specifically studied.

In this context, we sought to determine the prognostic capacity of laboratory values at presentation including WBC count, serum albumin and PLR in a series of patients with spinal metastases. We hypothesized that patients with low WBC count, low serum albumin and elevated PLR at presentation would be at increased risk of inferior outcomes including mortality, post-treatment complications and readmissions.

\section{Methods}

\section{Data Source and Inclusion Criteria}

This study was conducted using retrospective data obtained from the electronic medical records of patients treated at Brigham and Women's Hospital and Massachusetts General Hospital for spinal metastases between 2005 and 2017. Registry data from these centers have been successfully utilized in the past to develop predictive models for patients with cervical spine fractures ${ }^{25}$, epidural abscess ${ }^{26}$ and skeletal metastases ${ }^{2,14,18}$. Inclusion criteria consisted of patients aged 40-80 who received operative or non-operative management for spinal metastatic disease at one, or both, of our study centers. Non-operative management in this analysis consisted of any non-surgical treatment for cancer or malignancy related symptoms, including chemotherapy, radiation, immunotherapy and combinations thereof. In order to facilitate the development of a homogeneous cohort of patients with adequate representation of operative and non-operative cases we additionally restricted inclusion to individuals with a Deyo-modified Charlson Co-morbidity Index (CCI) count of conditions $<5$. Patients who received consultations without a definitive treatment plan, or who received 
definitive treatment at other centers, were excluded as were those with primary spinal tumors.

\section{Data Elements and Variable Definition}

We abstracted the medical records of patients identified as eligible and obtained age at the time of presentation, biologic sex, race (categorized as White or Non-White), Body Mass Index (BMI), CCI count (categorized as 1-2 vs 3-4), primary tumor (classified as breast, lung, or other tumors), location of the spinal metastases, presence of other metastases, vertebral body collapse or pathologic fracture at presentation and spinal canal compromise at presentation. Symptoms at presentation were also determined and categorized as asymptomatic, axial pain only and neurologic symptoms including radicular pain, claudication, myelopathy, paresis, paralysis, or bowel and/pr bladder dysfunction with presumed spinal etiology.

Ambulatory status at presentation was also recorded and defined as independently ambulatory, dependent/ambulatory with assistance (e.g. requiring use of a walker or cane) and non-ambulatory (e.g. bed or wheelchair bound greater than $50 \%$ of the time). For the purposes of this investigation, patients with documentation in their medical records of an Eastern Cooperative Oncology Group functional status of 3 or 4 were graded as nonambulatory. Laboratory values at presentation were also recorded including white blood cell count [WBC $\left.\left(\times 10^{3} / \mu \mathrm{L}\right)\right]$, albumin $(\mathrm{g} / \mathrm{dL})$ and platelet to lymphocyte ratio (PLR), an emerging marker of systemic inflammation ${ }^{18-20}$. Using previous literature as a guide ${ }^{17-24}$, we characterized normal laboratory values as: $\mathrm{WBC}$ count $>4.5$, albumin $>3.5$, and PLR $\leq 180$.

We recorded the type of treatment(s) received including chemotherapy, radiation and surgical intervention. We defined treatment as operative or non-operative, with any patient receiving surgical intervention (e.g. decompression, decompression and fusion) over the course of their care classified in the operative group, irrespective of other treatments they may have received. Patients treated only with a cement augmentation procedure were not considered in the surgical group in this analysis. Overall patient survival was assessed as well as mortality rates at 6-months and 1-year following presentation. Unplanned readmissions within 90-days of surgery, or the initiation of non-operative treatment for nonsurgical patients, were recorded as well as the development of complications (e.g. surgical site infections, skin and wound problems, shock, sepsis, delirium, venous thromboembolic events, as well as pulmonary, urinary, cardiovascular and cerebrovascular complications) assessed using a previously published algorithm ${ }^{2,9}$. We also surveyed patients for deterioration in ambulatory function with a final determination of ambulatory status determined at 6-months following presentation. Recognizing that the goals of treatment frequently include preservation of life and independence with activities of daily living, as well as palliation of pain, we developed a composite measure designated treatment failure which took into account patient survival and maintenance of ambulatory function at 6months following presentation. Individuals who were deceased at 6-months following presentation, or who were alive yet experienced a decline in ambulatory function, were designated treatment failures for the purposes of this investigation. 
The primary predictors in this study consisted of WBC count, albumin and PLR at presentation. Patients with WBC count $\leq 4.5$ were compared to those with counts $>4.5$. Serum albumin was evaluated using scores of 3.5 vs $>3.5$ and individuals with PLR $\leq 80$ were compared to patients with PLR $>180$. The primary outcome was length of survival. Mortality at 6-months and 1-year, complications, readmissions and the composite measure for treatment failure were considered secondary outcomes. Patient age, biologic sex, race, $\mathrm{CCI}$, primary tumor, location of spinal metastases, operative or non-operative treatment, year of presentation for treatment, vertebral body collapse and/or pathologic fracture at presentation, ambulatory status at presentation and symptoms at presentation were used as co-variates in all adjusted analyses.

\section{Statistical Analysis}

Initial unadjusted comparisons between the predictor variables and outcomes of interest were evaluated using Kaplan-Meier curves and the log-rank test to assess length of survival and chi-square testing for the secondary outcomes. Kaplan-Meier plots were also used to evaluate that the proportionality assumption was met such that multivariable Cox proportional hazard regression analysis could be used to analyze the relationship between laboratory values at presentation and length of survival, adjusting for confounders. ${ }^{28}$ Multivariable logistic regression was used to account for confounding in analyses related to secondary outcomes. Stepwise adjustment was performed accounting first for other laboratory values at presentation and then for all other co-variates in the study. The calibration of final logistic models was examined using the Hosmer-Lemeshow goodness of fit test. ${ }^{28}$ Statistical significance was set a-priori for variables with $p$-values $<0.05$ and Hazard Ratio (HR; Cox regression tests), or Odds Ratio (OR; logistic regression tests) with 95\% confidence intervals (CI) exclusive of 1.0 in the final adjusted models.

\section{Assessment of Stepwise Performance Using Scoring Rubric}

As a final assessment of the prognostic capacity of the laboratory values determined to be significant predictors in the statistical tests outlined above, we developed a scoring rubric based on performance in the final regression models. Score performance was assessed using multivariable logistic and Cox regression, accounting for all confounders. Internal validation was performed using a bootstrap simulation that consisted of sampling with replacement and 1,000 replications. The score was considered a useful prognostic tool for variables where a stepwise increase in score (e.g. 0 to1, 1 to 2 and 2 to 3 ) resulted in concomitant elevations in the odds of the outcome of interest that also retained statistical significance following adjusted testing and bootstrap validation.

This study received an exempt determination from our investigational review board prior to commencement. Study design and reporting are in accord with the Transparent Reporting of a multivariable prediction model for Individual Prognosis or Diagnosis (TRIPOD) statement. All analyses were performed using STATA v15.0 (STATA Corp., College Station, TX). 


\section{Results}

In the time period under study, we included 1,216 patients who met inclusion criteria. The average age of the population was 58.0 (SD 9.7), 50\% of the population was female and $84 \%$ were White (Table 1). Twenty-three percent of patients were diagnosed with lung cancer and 19\% had breast cancer (Appendix 1). Lung metastases were documented in 20\% of the cohort with liver metastases and additional bone metastases present in $23 \%$ and $49 \%$ of the population, respectively. Forty-one percent of patients presented with some degree of neurologic symptoms while $16 \%$ required assistive devices for ambulation and $7 \%$ were non-ambulators. WBC $\leq 4.5$ at presentation was recorded in $12 \%$, while serum albumin $₫ 3.5$ was present in $25 \%$ and PLR>180 documented in $52 \%$.

Thirty-seven percent of patients received a surgical intervention and $63 \%$ were treated nonoperatively. Overall, $30 \%$ of the cohort received chemotherapy and radiation, $19 \%$ were treated with chemotherapy alone and $10 \%$ radiation alone. Median survival for the cohort as a whole was 255 days (inter-quartile range 93-642 days), with 32\% mortality documented at 6 months and $47 \%$ mortality encountered at 1 -year following presentation. Complications occurred in $29 \%$ of the population and readmission events were documented in $35 \%$. Fortythree percent of patients met criteria to be designated a treatment failure.

Patients with PLR 480 , when compared to those with PLR>180 (heightened inflammatory state), were found to have lower rates of 6-month (22\% vs $42 \%)$ and 1-year mortality (34\% vs $58 \%$ ), as well as hospital readmission ( $32 \%$ vs $42 \%$ ). They were also less likely to experience treatment failure ( $32 \%$ vs $52 \%$ ) with complication rates relatively comparable ( $27 \%$ vs $32 \%$ ). Individuals with albumin $>3.5 \mathrm{~g} / \mathrm{dL}$, when compared to patients with albumin $3.5 \mathrm{~g} / \mathrm{dL}$, also experienced lower rates of 6-month (24\% vs 58\%) and 1-year mortality ( $39 \%$ vs $72 \%$ ) as well as reduced rates of treatment failure (35\% vs $68 \%$ ), complications ( $27 \%$ vs $36 \%$ ) and readmissions (35\% vs $42 \%$ ). Patients with WBC count

$\leq 4.5$, as compared to those with counts $>4.5$, had lower rates of 6-month mortality (25\% vs $35 \%$ ) and treatment failure (35\% vs $46 \%$ ), but comparable likelihoods of 1-year mortality (46\% vs $48 \%$ ) and complications (30\% vs $30 \%$ ). Raw readmission rates, meanwhile, were higher in this cohort ( $44 \%$ vs $35 \%)$.

\section{Influence of Laboratory Values at Presentation on Length of Survival}

Kaplan-Meier plots and log-rank testing revealed a significant association between PLR (Figure 1; $\mathrm{p}<0.001$ ) and serum albumin (Figure 2; $\mathrm{p}<0.001$ ) but not WBC count ( $\mathrm{p}=0.24$ ). Plots also revealed that Cox regression testing could be supported with unadjusted testing confirming the significant influence of PLR (HR 1.41; 95\% CI 1.20, 1.64) and serum albumin (HR 0.48; 95\% CI 0.41, 0.56), but no significant impact for WBC count (HR 1.14; $95 \%$ CI $0.92,1.41)$. These results were preserved after adjusted multivariable testing: PLR HR 1.53; 95\% CI 1.29, 1.80; $\mathrm{p}<0.001$, albumin HR 0.54; 95\% CI 0.45, 0.64; $<<0.001$, WBC count HR 1.08; $95 \%$ CI 0.86, 1.36; $\mathrm{p}=0.50$. 


\section{Influence of Laboratory Values on Secondary Outcomes}

Following adjusted multivariable regression testing, patients with PLR $\leq 80$, when compared to those with PLR>180 (heightened inflammatory state), were found to have lower odds of 6-month and 1-year mortality, as well as hospital readmission (Table 2). They were also less likely to experience treatment failure (OR $0.45 ; 95 \%$ CI $0.33,0.61 ; \mathrm{p}<0.001$ ). Individuals with albumin $>3.5 \mathrm{~g} / \mathrm{dL}$, when compared to patients with albumin $3.5 \mathrm{~g} / \mathrm{dL}$, were similarly found to have lower odds of 6-month and 1-year mortality as well as reduced odds of treatment failure (Table 3). There was no significant influence of serum albumin on the development of complications or readmissions. WBC count was only found to be significantly associated with 6-month mortality and the likelihood of readmissions (Appendix 2). There was no statistically significant evidence of poor model fit following Hosmer-Lemeshow testing among any of the final models (6-month mortality $\mathrm{p}=0.92 ; 1$ year mortality $\mathrm{p}=0.65$; complications $\mathrm{p}=0.06$; readmissions $\mathrm{p}=0.32$; treatment failure $\mathrm{p}=0.45$ ).

\section{Assessment of Stepwise Performance Using Scoring Rubric}

Based on their performance in the multivariable models, PLR and serum albumin were included in the final scoring rubric while WBC count was dropped from further consideration. Furthermore, based on the results of the multivariable models we determined that the scoring utility should weight serum albumin over PLR. Patients with PLR $>180$ were given a score of 1 , while those with serum albumin $3.5 \mathrm{~g} / \mathrm{dL}$ received a score of 2 . Patients with both characteristics received a score of 3 .

Kaplan-Meier testing confirmed a significant association between the scoring rubric and survival (Figure 3; $\mathrm{p}<0.001$ ). As compared to individuals with a score of 0 , significant stepwise increases in the likelihood of mortality were appreciated for patient with scores of 1 (HR 1.42; 95\% CI 1.17, 1.72; $\mathrm{p}<0.001$ ), 2 (HR 1.57; 95\% CI 1.14, 2.16; $\mathrm{p}=0.005$ ), and 3 (HR 2.76; 95\% CI 2.20, 3.48; $\mathrm{p}<0.001$ ) following adjusted Cox regression (Appendix 1). Likewise, significant stepwise elevations in the odds of 6-month and 1-year mortality, as well as treatment failure, were encountered in raw percentages (Appendix 2) as well as following multivariable logistic regression (Table 4; Appendix 3). The criterion of a stepwise effect was not experienced for either complications or readmissions (Table 4). These results were preserved in the internal validation performed using bootstrapping (Table 4).

\section{Discussion}

In this investigation, we found that PLR and serum albumin at presentation were significantly associated with survival, as well as 6-month and 1-year mortality and our composite measure for treatment failure in a large series of patients treated for spinal metastatic disease. Indeed, there also appears to be a synergistic effect between these laboratory measures, with stepwise significant increases in the likelihood of the adverse events we examined in individuals who presented with low serum albumin and an elevated PLR. While also significantly associated with the likelihood of complications and readmissions, a similar stepwise effect was not appreciated in the adjusted analyses regarding these outcomes, suggesting that albumin and PLR at presentation are less useful 
for forecasting these types of events. WBC count failed to meet our criteria as a useful prognostic measure regarding any of the outcomes considered.

Low serum albumin has been shown to be indicative of poor nutritional status and compromised wound healing in a variety of studies extending beyond the field of spinal metastatic disease. ${ }^{17,23,24}$ The link between low serum albumin and post-surgical morbidity, as well as mortality, has also been affirmed in patients treated operatively and nonoperatively for spinal metastases. ${ }^{2,9,14}$ It is important to recognize, however, that none of these prior works adjusted for other laboratory values at the time of presentation and, in some respects, estimations of serum albumin are proximate to the time of surgery and not necessarily presentation with spinal metastases. As a marker for a diminished health state, as well as a direct mediator of impaired wound healing, surgical site infections and venous thromboembolic disease, hypoalbuminemia likely plays an indirect role in potentiating the findings regarding diminished survival reported here.

Similarly, an elevated PLR has been described in prior literature as a herald of an increased systemic inflammatory state in patients with a variety of primary malignancies as well as skeletal metastases. ${ }^{18-22}$ Wang et al previously reported that elevated PLR was indicative of reduced disease-free survival, as well as overall survival, in patients with prostate cancer. ${ }^{19}$ Thio et al found that elevated PLR was associated with approximately 44\% mortality at 3months in patients treated for skeletal metastases. ${ }^{18}$ In the population of patients with spinal metastases, an elevated PLR may be indicative of generalized systemic dysfunction, a more aggressive subtype of primary tumor or advanced disease stage, increased osseous compromise or structural impairment, and/or higher degrees of neurologic compression. All of these factors may culminate in the increased odds of mortality, complications, readmissions and treatment failure encountered among individuals presenting with elevated PLR in this study.

This investigation is among the first we are aware of to evaluate the role of laboratory values at presentation, and specifically PLR, in prognosticating adverse events following operative or non-operative management of patients with spinal metastatic disease. The size of our cohort, as well as the fact that we restricted inclusion to a relatively homogeneous population treated in the modern period for spinal metastatic disease enhances the potential for translation of our study findings. The methodology employed here, the fact that we evaluated patients treated both operatively and non-operatively and adjusted for type of management in the multivariable analyses further enhances the capacity for generalization. In this context, we believe that there are several salient points that have immediate application to patient care and also suggest further lines of research. Foremost, clinicians can use our results to aid in initial counseling and discussions of natural history, treatment contingencies and anticipated outcomes at the time a patient presents with spinal metastases. In this setting, the potential for complications, readmission events, long-term survival and the risks and benefits of various treatment approaches can be addressed with more precision given an individual's serum albumin and PLR. Additionally, direct interventions intended to optimize nutritional status and reduce PLR prior to, or during, the initiation of treatment may minimize the risk for downstream adverse events. This suggests a line of future research whereby the efficacy of targeted interventions directed at improving these 
laboratory markers can be assessed. An additional opportunity for further research is suggested by the synergistic effect on adverse outcomes appreciated for patients with low serum albumin and elevated PLR. As serum albumin is a component of scoring systems such as the New England Spinal Metastasis Score (NESMS) ${ }^{9,14}$, it would be interesting to determine whether a modification that accounts for a patient's PLR would improve the accuracy of prognostic tools.

There are limitations associated with this research that should be recognized. First, this was a retrospective review of data collected from two tertiary academic centers that operate under a single corporate entity. Patient clustering and restricted treatment variation may occur as a result. We are also limited to variables regularly entered in the medical record for the purposes of patient care and potential errors in transcription cannot be quantified or addressed.

Restrictions on the clinical characteristics we have access to also impaired our capacity to utilize scoring systems such as the Tomita or Tokuhasi scales. This is an avenue of future research which we plan to address in a prospective study.

We further acknowledge the potential for selection and indication bias regarding treatment, although we attempted to control for this to the fullest extent possible by accounting for the performance of surgery in our adjusted analyses. Likewise, given the time frame for inclusion spans the years 2005-2017 there is added potential for surveillance bias with respect to mortality. We did include year of presentation for treatment in all multivariable analyses to account for this. In light of this fact, however, estimations regarding survival may be most applicable to patients in the first year following presentation. Our study also covers a time-period during which targeted molecular therapies and immunotherapies have emerged in treatment protocols. The impact of these therapies on lab values as a reflection of treatment response and their prognostic utility is not clear. Moreover, alterations in the WBC and PLR over the course of clinical care may be transient effects of treatment response and further investigation is needed to determine how these changes influence survival. Last, while our inclusion criteria were devised with the intent of assuring a representative sample of patients amenable to both operative and non-operative treatment, the fact that we limited consideration to patients aged $40-80$ and with comorbidities $<5$ means that the study findings may not be applicable to individuals outside of this age range, or with more extensive medical conditions. As a result, even in light of the bootstrap estimations, these findings require independent validation in an external population.

Despite these limitations, we believe that the size of our sample, clinical characteristics, methodologic approach and internal validation render the findings of this work readily applicable to clinical practice. At a minimum, individuals with low serum albumin and elevated PLR at the time of presentation should be advised regarding the implications of these laboratory markers for outcomes such as survival, irrespective of the treatment received. Secondarily, an effort should be made to optimize nutritional status and PLR prior to treatment, if practicable, in order to minimize the potential for adverse events associated with these parameters. 


\section{Supplementary Material}

Refer to Web version on PubMed Central for supplementary material.

\section{Acknowledgments}

Funding and Disclosure: This research was funded by a National Institutes of Health (NIH-NIAMS) grant (K23AR071464) to Dr. Schoenfeld. The views expressed in this article are those of the authors and do not necessarily reflect the position or policy of the NIH or the Federal government.

\section{References}

1. Rose PS, Buchowski JM. Metastatic disease in the thoracic and lumbar spine: Evaluation and management. J Am Acad Orthop Surg 2011;19: 37-48. [PubMed: 21205766]

2. Schoenfeld AJ, Leonard DA, Saadat E, Bono CM, Harris MB, Ferrone ML. Predictors of 30- and 90-Day Survival Following Surgical Intervention for Spinal Metastases: A Prognostic Study Conducted at Four Academic Centers. Spine (Phila Pa 1976). 2016;41: E503-9. [PubMed: 27064339]

3. Tabouret E, Cauvin C, Funetes S, Esterni B, Adetchessi T, Salem N, et al. Reassessment of scoring systems and prognostic factors for metastatic spinal cord compression. Spine J 2015;15: 944-950. [PubMed: 24120144]

4. Sailhan F, Prost S, Zairi F, Gille O, Pascal-Mousselard H, Bennis S, et al. Retrospective multicenter study by the French Spine Society of surgical treatment for spinal metastasis in France. Orthop Traumatol Surg Res. 2018730 pii: S1877-0568(18)30183-X. doi: 10.1016/j.otsr.2018.06.006. [Epub ahead of print].

5. Bauer HC, Wedin R. Survival after surgery for spinal and extremity metastases. Prognostication in 241 patients. Acta Orthop Scand 1995;66: 143-146. [PubMed: 7740944]

6. Barzilai O, McLaughlin L, Amato MK, Reiner AS, Ogilvie SQ, Lis E, et al. Predictors of quality of life improvement after surgery for metastatic tumors of the spine: prospective cohort study. Spine J. 2018;18:1109-1115. [PubMed: 29122701]

7. Paulino Pereira NR, Janssen SJ, van Dijk E, Harris MB, Hornicek FJ, Ferrone ML, et al. Development of a Prognostic Survival Algorithm for Patients with Metastatic Spine Disease. J Bone Joint Surg Am 2016;98: 1767-1776. [PubMed: 27807108]

8. Quraishi NA, Manoharan SR, Arealis G, Khurana A, Elsayed S, Edwards KL, et al. Accuracy of the revised Tokuhashi score in predicting survival in patients with metastatic spinal cord compression (MSCC). Eur Spine J 2013; 22 Suppl 1:S21-6. [PubMed: 23328875]

9. Schoenfeld AJ, Le HV, Marjoua Y, Leonard DA, Belmont PJ Jr, Bono CM, Harris MB. Assessing the utility of a clinical prediction score regarding 30-day morbidity and mortality following metastatic spine surgery: The New England Spinal Metastasis Score (NESMS). Spine J 2016;16: 482-490. [PubMed: 26409416]

10. Rades D, Huttenlocher S, Dunst J, Bajrovic A, Karstens JH, Rudat V, Schild SE. Matched pair analysis comparing surgery followed by radiotherapy and radiotherapy alone for metastatic spinal cord compression. J Clin Oncol 2010;28: 3597-3604. [PubMed: 20606090]

11. Bollen L, van der Linden YM, Pondaag W, Fiocco M, Pattynama BP, Marijnen CA, et al. Prognostic factors associated with survival in patients with symptomatic spinal bone metastases: a retrospective cohort study of 1,043 patients. Neuro Oncol. 2014;16: 991-8. [PubMed: 24470544]

12. Bollen L, Wibmer C, Van der Linden YM, Pondaag W, Fiocco M, Peul WC, et al. Predictive Value of Six Prognostic Scoring Systems for Spinal Bone Metastases: An Analysis Based on 1379 Patients. Spine 2016;41: E155-E162. [PubMed: 26866742]

13. Dea N, Versteeg A, Fisher C, Kelly A, Hartig D, Boy M, et al. Adverse events in emergency oncological spine surgery: A prospective analysis. J Neurosurg Spine 2014;21: 698-703. [PubMed: 25147976] 
14. Shi DD, Chen YH, Lam TC, Leonard D, Balboni TA, Schoenfeld A, et al. Assessing the utility of a prognostication model to predict 1-year mortality in patients undergoing radiation therapy for spinal metastases. Spine J 2018;18: 935-940. [PubMed: 29031992]

15. Tomita K, Kawahara N, Kobayashi T, Yoshida A, Murakami H, Akamaru T. Surgical strategy for spinal metastases. Spine 2001;26: 298-306. [PubMed: 11224867]

16. Tokuhashi Y, Matsuzaki H, Toriyama S, Kawano H, Ohsaka S. Scoring system for the preoperative evaluation of metastatic spine tumor prognosis. Spine 1990;15: 1110-1113. [PubMed: 1702559]

17. Schoenfeld AJ, Carey PA, Cleveland AW III, Bader JO, Bono CM. Patient factors, comorbidities and surgical characteristics that increase mortality and complication-risk following spinal arthrodesis: A prognostic study based on 5,887 patients. Spine J 2013;10: 1171-1179.

18. Thio QCBS, Goudriaan WA, Janssen SJ, Paulino Pereira NR Sciubba DM, Rosovksy RP, Schwab JH. Prognostic role of neutrophil-to-lymphocyte ratio and platelet-to-lymphocyte ratio in patients with bone metastases. Br J Cancer. 2018; 119: 737-743. [PubMed: 30116026]

19. Wang J, Zhou X, He Y, Chen X, Liu N, Ding Z, Li J. Prognostic role of platelet to lymphocyte ratio in prostate cancer: A meta-analysis. Medicine (Baltimore). 2018;97: e12504. [PubMed: 30290605]

20. Lee BM, Chung SY, Chang JS, Lee KJ, Seong J. The Neutrophil-Lymphocyte Ratio and PlateletLymphocyte Ratio Are Prognostic Factors in Patients with Locally Advanced Pancreatic Cancer Treated with Chemoradiotherapy. Gut Liver. 2018;12: 342-352. [PubMed: 29409306]

21. Furuyama T, Onohara T, Yamashita S, Yoshiga R, Yoshiya K, Inoue K, et al. Prognostic factors of ulcer healing and amputation-free survival in patients with critical limb ischemia. Vascular. 2018: 1708538118786864. doi: 10.1177/1708538118786864. [Epub ahead of print].

22. Cho U, Park HS, Im SY, Yoo CY, Jung JH, Suh YJ, Choi HJ. Prognostic value of systemic inflammatory markers and development of a nomogram in breast cancer. PLoS One. 2018;13: e0200936. [PubMed: 30048474]

23. Dickhaut SC, DeLee JC, Page CP. Nutritional status: importance in predicting wound healing in amputations. J Bone Joint Surg Am 1984;66:71-5. [PubMed: 6690446]

24. Klein JD, Hey LA, Yu CS, et al. Perioperative nutrition and postoperative complications in patients undergoing spinal surgery. Spine 1996;21:2676-82. [PubMed: 8961455]

25. Schoenfeld AJ, Bono CM, Reichmann WM, Warholic N, Wood KB, Losina E, et al. Type II odontoid fractures of the cervical spine: do treatment type and medical comorbidities affect mortality in elderly patients? Spine 2011;36: 879-85. [PubMed: 21217435]

26. Kim SD, Melikian R, Ju KL, Zurakowski D, Wood KB, Bono CM, Harris MB. Independent predictors of failure of nonoperative management of spinal epidural abscesses. Spine J. 2014;14: 1673-9. [PubMed: 24373683]

27. Deyo RA, Cherkin DC, Ciol MA. Adapting a clinical comorbidity index for use with ICD-9-CM administrative databases. J Clin Epidemiol. 1992;45: 613-9. [PubMed: 1607900]

28. Long JS, Freese J. Regression models for categorical dependent variables using STATA. 2nd Edition. College Station, TX: STATA Press, 2006.

29. Collins GS, Reitsma JB, Altman DG, Moons KG. Transparent Reporting of a multivariable prediction model for Individual Prognosis or Diagnosis (TRIPOD): the TRIPOD statement. Ann Int Med 2015;162: 55-63. [PubMed: 25560714] 


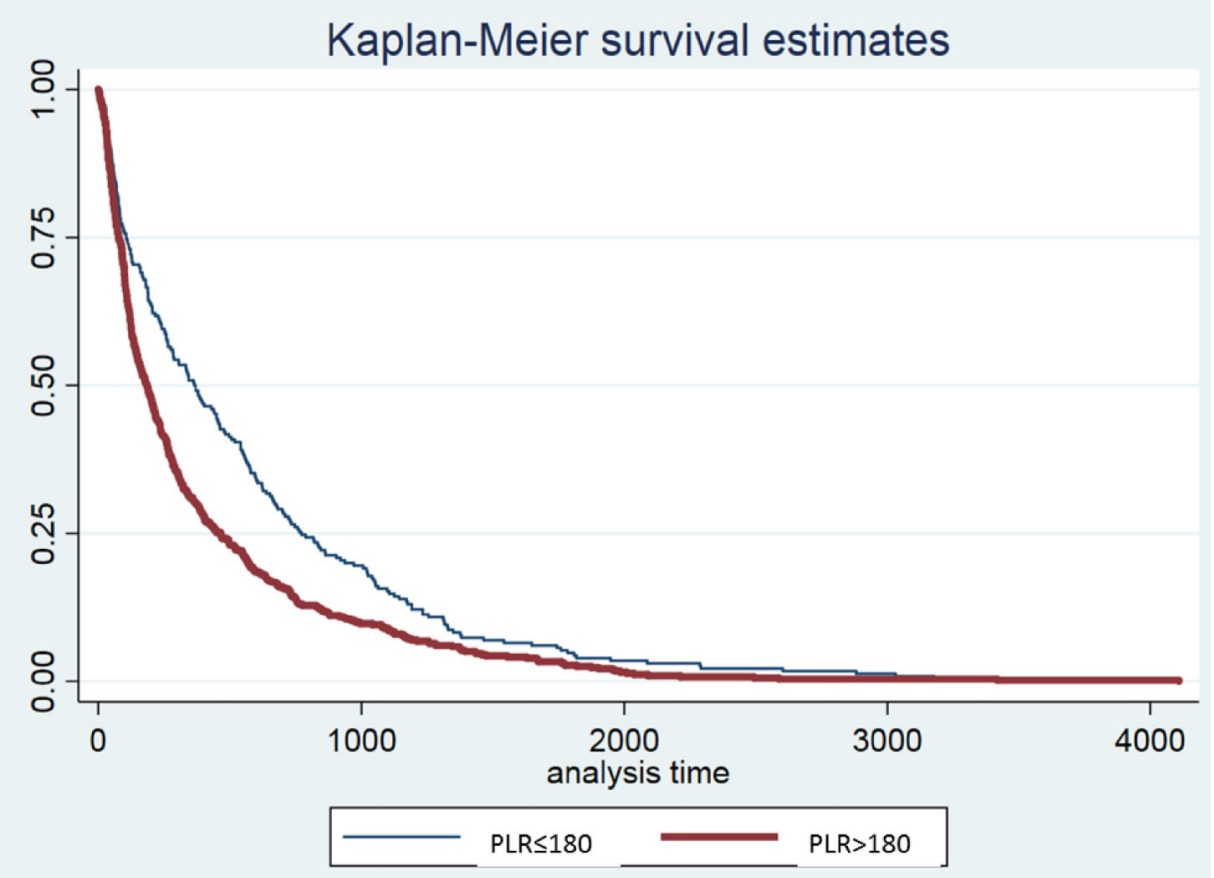

Figure 1. 


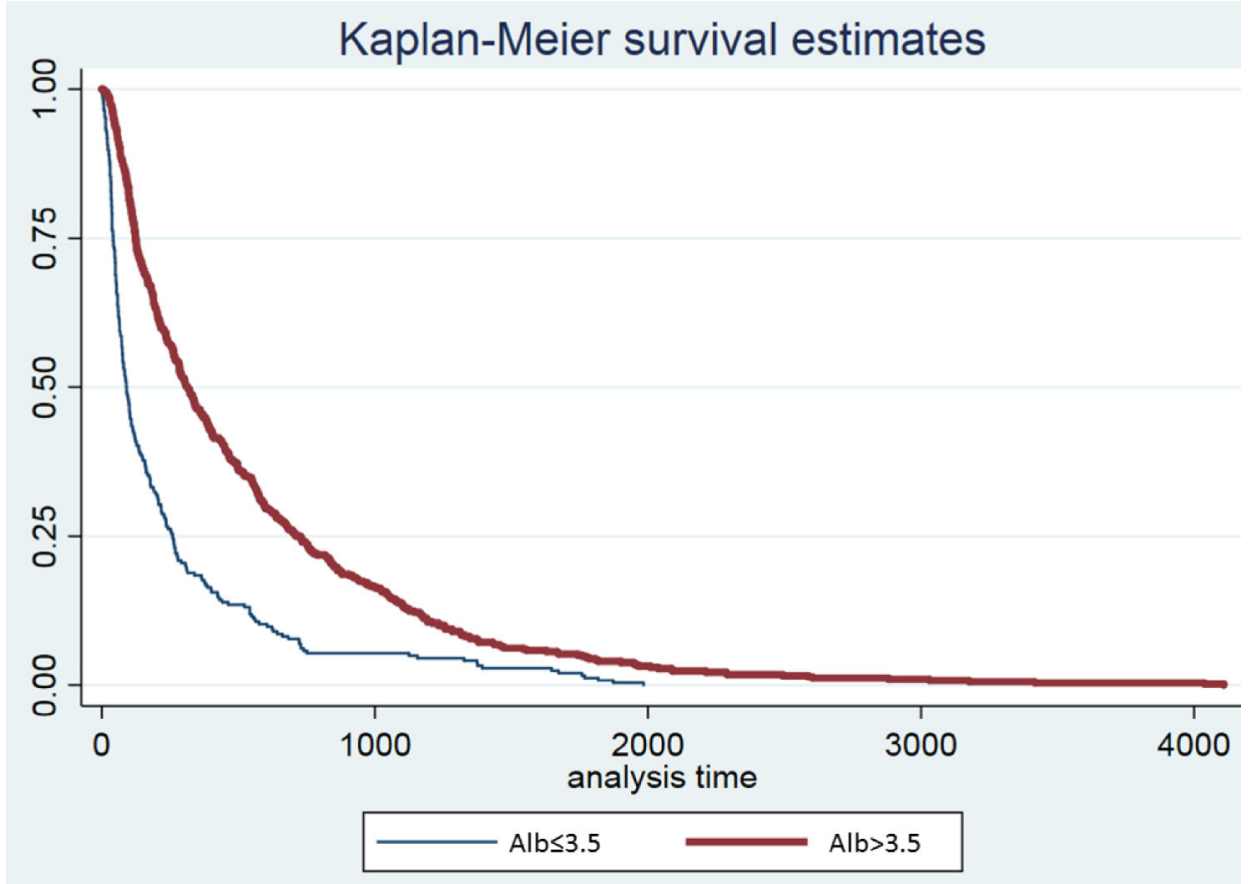

Figure 2. 


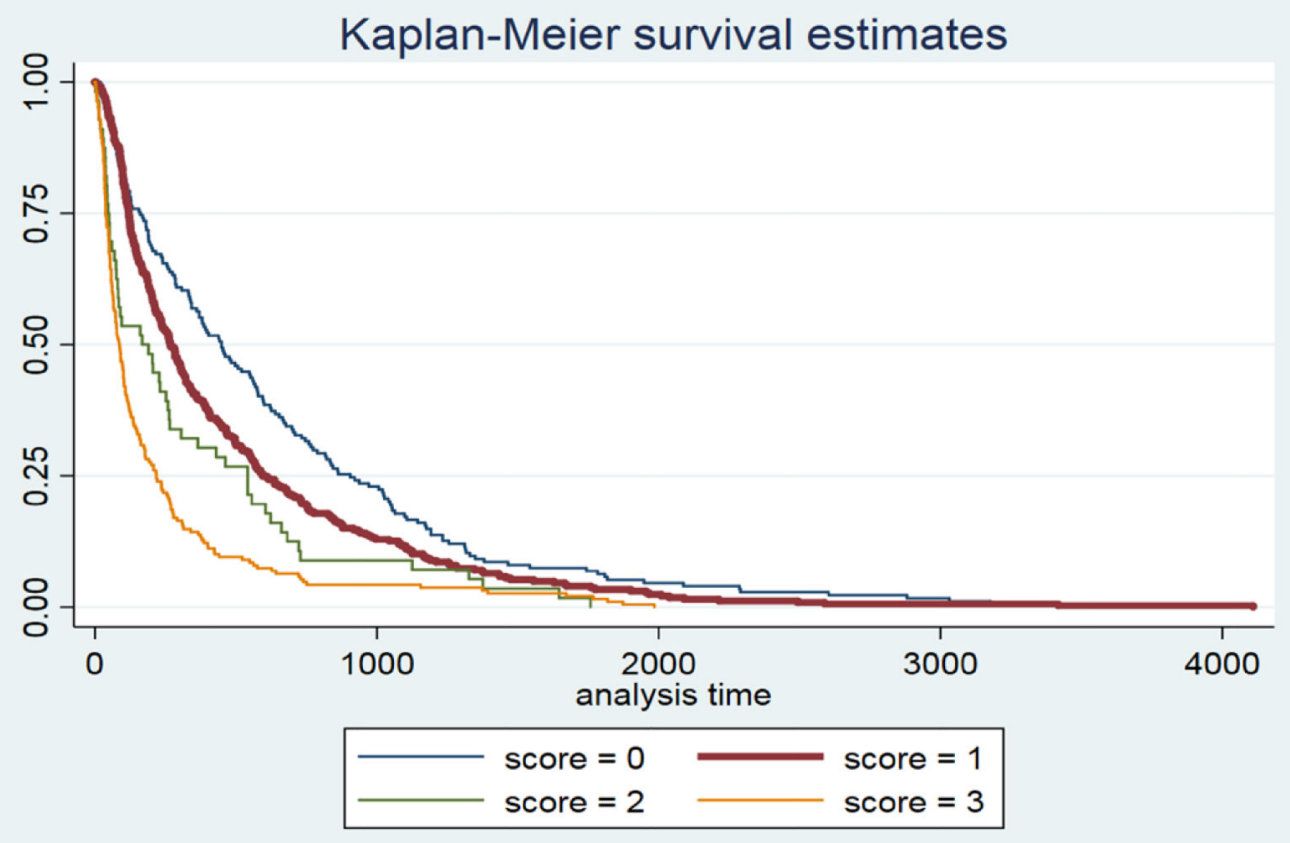

Figure 3. 
Table 1.

Demographic characteristics of patients treated for spinal metastases between 2005-2017. There were 1,216 patients in the total sample from which the proportions below are derived.

\begin{tabular}{|c|c|}
\hline Characteristics & $\mathbf{N}(\%)^{@}$ \\
\hline Age (mean, SD) & $58.0(9.7)$ \\
\hline Female Sex & $608(50)$ \\
\hline White & $1,016(84)$ \\
\hline Body Mass Index (mean, SD) & $27.1(6.0)$ \\
\hline \multicolumn{2}{|l|}{ Number of Comorbidities } \\
\hline $1-2$ & $743(61)$ \\
\hline $3-4$ & $473(39)$ \\
\hline \multicolumn{2}{|l|}{ Primary Cancer } \\
\hline Breast & $231(19)$ \\
\hline Lung & $279(23)$ \\
\hline Other & $706(58)$ \\
\hline Albumin $3.5 \mathrm{~g} / \mathrm{dL}^{\#}$ & $303(25)$ \\
\hline WBC Count $\leq 4.5^{\$}$ & $145(12)$ \\
\hline Platelet to Lymphocyte Ratio $>180^{\&}$ & $637(52)$ \\
\hline Lung Metastases & $247(20)$ \\
\hline Liver Metastases & $285(23)$ \\
\hline Additional Bone Metastases & $590(49)$ \\
\hline Vertebral Body Collapse/Pathologic Fracture & $589(48)$ \\
\hline Spinal Canal Compromise & $605(50)$ \\
\hline \multicolumn{2}{|l|}{ Ambulatory Status at Presentation } \\
\hline Independent Ambulator & $939(77)$ \\
\hline Ambulatory with Assistance & $192(16)$ \\
\hline Non-ambulator & $83(7)$ \\
\hline \multicolumn{2}{|l|}{ Symptoms at Presentation } \\
\hline Axial Pain/Asymptomatic & $722(59)$ \\
\hline Neurologic Symptoms & $494(41)$ \\
\hline \multicolumn{2}{|l|}{ Treatment } \\
\hline Operative & $448(37)$ \\
\hline Non-Operative & $768(63)$ \\
\hline \multicolumn{2}{|l|}{ @ - Except where otherwise specified. } \\
\hline
\end{tabular}

Spine J. Author manuscript; available in PMC 2021 January 01. 
Table 2.

Estimates of the effect of platelet/lymphocyte ratio (PLR) on outcomes among patients treated for spinal metastases (2005-2017). Results are presented as odds ratio (OR) with 95\% Confidence Interval (CI) for patients with PLR $\leq 80$ compared to those with PLR $>180$ as the referent. ORs and 95\% CI exclusive of 1.0 were considered significant (bold text).

\begin{tabular}{|c|c|c|c|c|c|}
\hline Effect of Surgery & $\begin{array}{c}\text { 6-month } \\
\text { Mortality OR } \\
\text { (95\% CI) }\end{array}$ & $\begin{array}{l}\text { 1-year Mortality } \\
\text { OR }(95 \% \text { CI) }\end{array}$ & $\begin{array}{c}\text { Treatment } \\
\text { Failure OR (95\% } \\
\text { CI) }\end{array}$ & $\underset{(95 \% \text { CI })}{\text { Complications OR }}$ & $\underset{(95 \% \mathrm{CI})}{\text { Readmissions OR }}$ \\
\hline Unadjusted & $0.40(0.29,0.53)$ & $0.36(0.28,0.48)$ & $0.42(0.32,0.55)$ & $0.78(0.59,1.04)$ & $0.65(0.49,0.85)$ \\
\hline $\begin{array}{l}\text { Adjusted for } \\
\text { Laboratory Values }\end{array}$ & $0.45(0.33,0.61)$ & $0.40(0.30,0.53)$ & $0.47(0.36,0.63)$ & $0.82(0.61,1.09)$ & $0.67(0.51,0.88)$ \\
\hline $\begin{array}{l}\text { Adjusted for } \\
\text { Laboratory Values and } \\
\text { Clinical } \\
\text { Characteristics }\end{array}$ & $0.42(0.30,0.58)$ & $0.37(0.27,0.50)$ & $0.45(0.33,0.61)$ & $0.85(0.63,1.16)$ & $0.67(0.50,0.90)$ \\
\hline
\end{tabular}

Ref - referent 
Table 3.

Estimates of the effect of albumin on outcomes among patients treated for spinal metastases (2005-2017). Results are presented as odds ratio (OR) with $95 \%$ Confidence Interval (CI) for patients with albumin $>3.5 \mathrm{~g} / \mathrm{dL}$ compared to those with albumin $3.5 \mathrm{~g} / \mathrm{dL}$ as the referent. ORs and $95 \% \mathrm{CI}$ exclusive of 1.0 were considered significant (bold text).

\begin{tabular}{|c|c|c|c|c|c|}
\hline & $\begin{array}{c}\text { 6-month } \\
\text { Mortality OR } \\
\text { (95\% CI) }\end{array}$ & $\begin{array}{l}\text { 1-year Mortality } \\
\text { OR (95\% CI) }\end{array}$ & $\begin{array}{c}\text { Treatment } \\
\text { Failure OR (95\% } \\
\text { CI) }\end{array}$ & $\underset{(95 \% \text { CI })}{\text { Complications OR }}$ & $\begin{array}{c}\text { Readmissions OR } \\
(95 \% \mathrm{CI})\end{array}$ \\
\hline Unadjusted & $0.22(0.17,0.30)$ & $0.25(0.19,0.34)$ & $0.25(0.19,0.34)$ & $0.67(0.50,0.89)$ & $0.75(0.57,0.98)$ \\
\hline $\begin{array}{l}\text { Adjusted for } \\
\text { Laboratory Values }\end{array}$ & $0.24(0.18,0.32)$ & $0.27(0.20,0.36)$ & $0.27(0.20,0.36)$ & $0.68(0.51,0.91)$ & $0.75(0.57,0.99)$ \\
\hline $\begin{array}{l}\text { Adjusted for } \\
\text { Laboratory Values } \\
\text { and Clinical } \\
\text { Characteristics }\end{array}$ & $0.32(0.23,0.44)$ & $0.35(0.25,0.49)$ & $0.33(0.24,0.45)$ & $0.74(0.54,1.02)$ & $0.85(0.63,1.16)$ \\
\hline
\end{tabular}

Ref - referent 
Table 4.

Estimates of the effect of possessing zero, one, two or all three adverse lab parameters (PLR $\leq 80$, albumin $\$ 3.5 \mathrm{~g} / \mathrm{dL}$ and/or WBC Count $\leq 4.5$ ) on outcomes among patients treated for spinal metastases (2005-2017). Results are presented as odds ratio (OR) with 95\% Confidence Interval (CI). ORs and 95\% CI exclusive of 1.0 were considered significant (bold text).

\begin{tabular}{|c|c|c|c|c|c|}
\hline & $\begin{array}{c}\text { 6-month Mortality } \\
\text { OR }(95 \% \text { CI })^{*}\end{array}$ & $\begin{array}{c}\text { 1-year Mortality } \\
\text { OR }(95 \% \text { CI })^{*}\end{array}$ & $\begin{array}{c}\text { Treatment Failure } \\
\text { OR }(95 \% \text { CI })^{*}\end{array}$ & $\begin{array}{c}\text { Complications OR } \\
(95 \% \text { CI })\end{array}$ & $\begin{array}{c}\text { Readmissions OR } \\
(95 \% \mathrm{CI})\end{array}$ \\
\hline \multicolumn{6}{|l|}{ Unadjusted } \\
\hline Zero & Ref & Ref & Ref & Ref & Ref \\
\hline One & $1.92(1.32,2.80)$ & $2.38(1.72,3.31)$ & $1.87(1.34,2.61)$ & $1.43(1.01,2.04)$ & $1.82(1.31,2.54)$ \\
\hline Two & $3.27(1.84,5.80)$ & $3.47(2.00,6.00)$ & $2.89(1.67,4.99)$ & $2.47(1.41,4.31)$ & $2.74(1.59,4.71)$ \\
\hline Three & $9.26(6.05,14.20)$ & $9.89(6.43,15.21)$ & $7.92(5.23,11.97)$ & $1.75(1.17,2.61)$ & $1.82(1.24,2.68)$ \\
\hline \multicolumn{6}{|l|}{$\underline{\text { Adjusted }}$} \\
\hline Zero & Ref & Ref & Ref & Ref & Ref \\
\hline One & $1.92(1.28,2.89)$ & $2.55(1.78,3.66)$ & $1.94(1.35,2.78)$ & $1.53(1.05,2.22)$ & $1.98(1.39,2.82)$ \\
\hline Two & $2.25(1.18,4.26)$ & $2.79(1.53,5.11)$ & $2.53(1.39,4.62)$ & $2.84(1.54,5.24)$ & $3.06(1.69,5.56)$ \\
\hline Three & $7.28(4.57,11.61)$ & $8.37(5.22,13.41)$ & $6.82(4.35,10.70)$ & $1.64(1.05,2.56)$ & $1.65(1.08,2.52)$ \\
\hline \multicolumn{6}{|l|}{$\begin{array}{l}\text { Bootstrap } \\
\text { Procedure }\end{array}$} \\
\hline Zero & Ref & Ref & Ref & Ref & Ref \\
\hline One & $1.92(1.31,2.81)$ & $2.38(1.71,3.33)$ & $1.87(1.34,2.61)$ & $1.43(1.01,2.02)$ & $1.82(1.30,2.55)$ \\
\hline Two & $3.27(1.78,5.99)$ & $3.47(1.98,6.07)$ & $2.89(1.62,5.15)$ & $2.47(1.39,4.39)$ & $2.74(1.59,4.70)$ \\
\hline Three & $9.26(5.95,14.42)$ & $9.89(6.33,15.44)$ & $7.92(5.12,12.25)$ & $1.75(1.17,2.62)$ & $1.82(1.26,2.64)$ \\
\hline
\end{tabular}

Ref - referent. Variables with an

*

exhibit the desired stepwise function within the scoring system. 